\title{
Noms de partis et messages politiques : le cas des partis politiques kurdes légaux en Turquie
}

Party names and political messages: the case of legal Kurdish political parties in Turkey

Nombres de partidos y mensajes políticos: el caso de los partidos políticos legales kurdos en Turquía

\section{Salih Akin}

\section{OpenEdition}

\section{Journals}

Édition électronique

URL : https://journals.openedition.org/mots/25246

DOI : $10.4000 /$ mots. 25246

ISSN : 1960-6001

Éditeur

ENS Éditions

\section{Édition imprimée}

Date de publication : 11 juillet 2019

Pagination : 91-107

ISBN : 979-10-362-0170-7

ISSN : 0243-6450

\section{Référence électronique}

Salih Akin, « Noms de partis et messages politiques : le cas des partis politiques kurdes légaux en Turquie », Mots. Les langages du politique [En ligne], 120 | 2019, mis en ligne le 01 janvier 2022, consulté le 23 avril 2022. URL : http://journals.openedition.org/mots/25246 ; DOI : https://doi.org/10.4000/ mots. 25246 


\section{Noms de partis et messages politiques : le cas des partis politiques kurdes légaux en Turquie}

Après avoir milité durant de nombreuses années au sein des partis politiques condamnés à la clandestinité, les secteurs militants de la communauté kurde de Turquie ont créé leurs premiers partis politiques légaux au début des années 1990 en utilisant quelques marges de manœuvre démocratiques. Seize partis politiques ont vu le jour depuis, mais peu d'entre eux ont échappé aux procès politiques intentés par l'État turc, qui se sont soldés par leur dissolution et, parfois, l'incarcération de leurs dirigeants. Le cas le plus symbolique est sans doute celui du DEP (Parti de la démocratie) fondé en 1991 et dissous en 1994, après l'arrestation et la condamnation à 15 ans d'emprisonnement de ses dix députés. Dans un pays politiquement polarisé et traversant de nos jours une grave crise politique en matière de fonctionnement démocratique et de libertés fondamentales, nommer un parti est tout sauf anodin. Les noms des partis, comme la mise en mots de leurs objectifs, sont en effet soumis à des contraintes légales qui restreignent considérablement le cadre de leurs discours comme celui de leurs activités. Ces contraintes conduisent les formations politiques à élaborer des stratégies discursives pour contourner les restrictions légales sur le nom des partis et la mise en mots de leurs objectifs.

Dans cette contribution, nous examinerons le cas des noms des partis kurdes légaux en Turquie. Pour cela, nous allons donner, dans un premier temps, les principaux repères de la question kurde et des mouvements politiques kurdes légaux en Turquie. Nous discuterons également les contraintes officielles et politiques qui pèsent sur la nomination des partis et leurs activités à la lumière de la loi no 2820 sur le statut des partis politiques. Nous analyserons dans un deuxième temps le nom de dix-sept partis politiques kurdes légaux, à la fois du point de vue structurel et lexico-sémantique. L'analyse mettra en lumière la façon dont les contraintes légales sur la dénomination des partis les conduisent à imaginer et à déployer des stratégies fondées essentiellement sur l'évitement et l'implicite. 


\section{La question kurde en Turquie}

L'un des objectifs principaux des partis politiques kurdes légaux est d'apporter des solutions à la question kurde en Turquie, qui s'enracine dans le démembrement de l'Empire ottoman et dans la construction d'États-nations sur ses ruines au lendemain de la Première Guerre mondiale (Bozarslan, 1997; Yilmaz, 2013). Après le génocide des Arméniens en 1915 et l'expulsion des Grecs en 1922, l'Empire abritait encore de nombreuses composantes ethniques et linguistiques (Arabes, Abkhazes, Assyriens, Bulgares, Kurdes, Tchétchènes, Tcherkesses, etc.). L'État turc, fondé en 1923, s'est lancé à cette époque dans une politique d'homogénéisation du fondement multiethnique et multilingue de l'Empire ottoman et s'est donné pour objectif d'assimiler les minorités à la langue et à la culture turques ${ }^{1}$. La population non turque la plus importante du point de vue démographique est constituée des Kurdes, peuple dispersé dans d'autres pays comme l'Irak, l'Iran et la Syrie².

L'un des piliers de cette politique d'homogénéisation ethnique et linguistique fut la dénégation de l'existence des Kurdes en Turquie. Dans ce sens, un décret-loi publié le 3 mars 1924 interdit les écoles, les associations et les publications kurdes, et en même temps l'emploi des termes Kurde et Kurdistan dans toutes les publications comme dans l'usage quotidien (Bozarlsan, 1997). Un véritable tabou linguistique fut institué au sujet de l'usage de ces termes, ce qui a posé, comme on l'imagine, d'importantes difficultés linguistiques aux autorités turques pour faire référence au peuple, au territoire et à la langue kurdes (Akin, 1995). Déniés dans leur existence, les Kurdes de Turquie ont été aussi privés, jusqu'aux années 1990, de tout droit culturel et linguistique, comme celui d'une éducation dans leur langue ou d'écouter de la musique en kurde (Skutnabb-Kangas, Phillipson, 1999).

\section{La mobilisation kurde au sein de partis politiques turcs}

Cette répression linguistique et culturelle a conduit les Kurdes à s'organiser dans un premier temps en mouvements politiques clandestins et à entreprendre plusieurs révoltes et soulèvements de 1925 à 1938 (Bozarslan, 1997). L'échec politique et militaire des soulèvements oblige les mouvements à envisager d'autres moyens de lutte. L'ouverture démocratique en Turquie dans les

1. Malgré la politique d'assimilation mise en place depuis la fondation de l'État turc, le projet Joshua recense 68 groupes ethnolinguistiques dans la Turquie contemporaine, https://joshuaproject.net/countries/TU (consulté le 08/03/2019).

2. Selon les estimations non officielles, il y aurait entre 35 millions et 45 millions de Kurdes. «La population kurde », Institut kurde de Paris, https://www.institutkurde.org/info/la-populationkurde-1232550992 (consulté le 08/03/2019). 
années 1960 et le passage à un régime multipartite constituent les deux facteurs principaux qui ont permis à une partie des Kurdes de s'organiser, dans un second temps, au sein de mouvements politiques légaux, alors que d'autres ont choisi de poursuivre le combat pour les droits culturels et linguistiques au sein de partis clandestins.

Cet engagement politique dans un cadre légal est d'abord exercé au sein d'organisations turques de gauche et d'extrême gauche. Le Türkiye İşçi Partisi (TIP) «Parti ouvrier de Turquie », fondé en 1961, est le premier parti légal de la gauche turque à intégrer des cadres kurdes.

L'idéologie et le programme du parti, qui regroupait des secteurs progressistes de la société turque, constituaient un changement radical par rapport à ceux des partis traditionnels turcs. Prenant ses distances avec l'idéologie kémaliste 3 , le TIP prônait une lutte pour la démocratie et le socialisme, qui permettrait de reconnaître les droits nationaux des Kurdes de Turquie. Cette approche démocratique de la question kurde a attiré dans ses rangs nombre d'intellectuels et activistes kurdes (Akin, 1996).

Cette première socialisation politique dans un cadre légal sera poursuivie, après la dissolution du TIP en 1971 par la Cour constitutionnelle, au sein de quelques autres partis politiques turcs, fondés après le coup d’État militaire de 1980. Le Sosyal Demokrat Halkçı Parti (SHP) «Parti sociodémocrate et populaire » fondé en 1985 a reçu ainsi un soutien significatif des cadres et électeurs kurdes (Yilmaz, 2013, p. 150).

\section{L'émergence de partis politiques kurdes légaux sur la scène politique turque}

Cependant, la gauche turque légale, avec ses bases kémalistes, ses penchants nationalistes et une approche essentiellement électoraliste, se révèle incapable de proposer une solution démocratique à la question kurde, qui pourrait se traduire par l'attribution des droits linguistiques et culturels élémentaires à la population kurde de Turquie. L'expulsion du Parti sociodémocrate de sept députés kurdes, accusés par leur parti d'avoir participé à une conférence intitulée «L'identité nationale kurde et les droits de l'homme » et organisée à Paris en 1989, conduit le mouvement kurde à chercher des solutions alternatives de lutte sur le plan légal. C'est dans ce contexte que naît le Halkın Emek Partisi (HEP) «Parti du travail du peuple», qui constitue la première expérience de parti légal kurde. Fondé en 1990 en pleine guérilla armée du Parti des travailleurs du Kurdistan (PKK) et politique de répression qui s'en est suivie avec la

3. L’idéologie kémaliste est constituée de six principes de conduite des affaires publiques : républicanisme, nationalisme, laïcisme, populisme, étatisme et réformisme, ou esprit révolutionnaire (Schmid, 2017). Pour une discussion des évolutions récentes de l'idéologie kémaliste, voir Deren, 2005 . 
disparition de milliers d'intellectuels, journalistes, écrivains ou simples militants, le HEP symbolisa la montée en puissance des revendications identitaires kurdes en Turquie. Cette première expérience bouleverse la scène politique turque, où se forme désormais un bloc kurde portant publiquement et légalement la question kurde au Parlement et dans l'opinion publique. Cette volonté de débattre publiquement d'une question épineuse sera censurée par la Cour constitutionnelle, qui dissout le parti en 1993 pour violation des statuts des partis politiques. Dès lors, commence un processus politico-judiciaire qui obligera le mouvement kurde à créer des « partis de réserve » (Yilmaz, 2013) en prévision de possibles poursuites judiciaires en dissolution. Mis à part deux partis, le Demokratik Bölgeler Partisi (DBP) «Parti des régions démocratiques », et le Halklarin Demokratik Partisi (HDP) «Parti démocratique des peuples», huit autres partis poursuivant l'héritage du HEP ont été dissous l'un après l'autre par la Cour constitutionnelle4. Alors que le DBP, «parti de réserve », n'a participé encore à aucune élection, le HDP, fondé en 2012, est représenté au Parlement turc avec 58 députés 5 . Aucun procès n'est engagé pour l'instant contre le DBP. En revanche, contrairement aux usages, c'est un parti nationaliste turc, Vatan Partisi «Parti de la patrie», et non la Cour constitutionnelle, qui a lancé en 2016 un procès en dissolution contre le HDP, dont le président Selahattin Demirtaş, ainsi que dix députés, ont été arrêtés et incarcérés la même année ${ }^{6}$. Accusé des chefs de "soutien et propagande du PKK», M. Demirtaş encourt une peine de prison de 142 ans.

Parallèlement à la tradition politique initiée par le HEP et poursuivie de nos jours par le HDP, d'autres partis politiques ont vu le jour, reflétant différentes sensibilités politiques de la société kurde7. Avant d'analyser les stratégies de nomination des partis, examinons les contraintes légales sur leurs statuts et leurs noms.

4. Demokrasi Partisi «Parti de la démocratie » créé en 1991, dissous en 1994; Özgürlük ve Demokrasi Partisi «Parti de la liberté et de la démocratie», 1992-1993; Özgürlük ve Eşitlik Partisi «Parti de la liberté et de l'égalité », 1992-1993; Halkın Demokrasi Partisi «Parti démocratique du peuple», 1994-2003; Demokratik Halk Partisi « Parti du peuple démocratique», 1997-2005; Demokratik Toplum Partisi «Parti de la société démocratique», 1997-2005; Barış ve Demokrasi Partisi «Parti de la paix et la démocratie», 2008-2014.

5. Aux élections législatives anticipées du 24 juin 2018 , le HDP a obtenu $11 \%$ des voix.

6. "Liste des prisons des députés du HDP», http://fr.hdpeurope.com/?p=3813 (consulté le 08/03/2019).

7. Il s'agit des partis Hak ve özgürlükler Partisi «Parti des droits et des libertés» (HAK-PAR), créé en 2002; Katılımcı Demokrasi Partisi «Parti de la démocratie participative » (KADEP), créé en 2006 ; Hür Dava Partisi «Parti de la cause libre » (HÜDA-PAR) créé en 2012 ; Türkiye Kürdistan Demokrat Partisi «Parti démocratique du Kurdistan de Turquie» (TKDP), créé en 2014 ; Kürdistan Özgürlük Partisi «Parti de la liberté du Kurdistan » (PAK), créé en 2014 et le Kürdistan Sosyalist Partisi «Parti socialiste du Kurdistan» (PSK), créé en 2016. Des procès sont en cours à la Cour constitutionnelle pour la dissolution du TDKP, PAK et PSK. 


\section{Les contraintes officielles sur les statuts et le nom des partis politiques}

Les libertés, les droits et les devoirs des partis sont strictement encadrés par la loi n 2820 (publiée au Journal officiel de la République de Turquie le 24/04/1983) sur le statut des partis politiques. Une série de contraintes limite les objectifs et les activités de ceux-ci. Dans une section intitulée «Prévention de création des minorités ", l'article 81 de la loi stipule que les partis politiques:

a) ne peuvent affirmer qu'il existe sur le territoire de la République de Turquie des minorités fondées sur une différence nationale ou religieuse, culturelle ou confessionnelle, raciale ou linguistique;

b) ne peuvent avoir pour objectif de mener des activités visant à saper l'unité nationale en créant des minorités sur le territoire de la République de Turquie par la protection, le développement et la diffusion d'une langue et d'une culture autres que la langue et la culture turques;

c) ne peuvent utiliser une langue autre que le turc dans la rédaction et la publication de leurs statuts et leur programme, dans leurs congrès, réunions, meetings et dans leur propagande; utiliser et distribuer des pancartes, enseignes, disques, bandes sonores et visuelles, brochures et déclarations écrits en d'autres langues que le turc; et ne peuvent pas rester insensibles à ce que ces actions soient entreprises pardes tiers [...].

Dans une autre section intitulée «Interdiction du régionalisme et du racisme», l'article 82 précise que «les partis politiques ne peuvent pas avoir pour objectif de favoriser le régionalisme et le racisme dans le pays qui est un ensemble indivisible et ne peuvent mener des activités dans cet objectif». Énonçant des contraintes sur les objectifs et les activités des partis, les articles 81 et 82 s'inscrivent dans une modalité négative, de l'ordre du « ne pas faire ». Ils contraignent les partis politiques à faire abstraction, dans leurs activités, de l'épineuse question des altérités ethnolinguistiques en Turquie, altérités qui se trouvent englobées dans une palette hétéroclite.

Cependant, par l'interdiction, le texte législatif affirme ce qu'il cherche précisément à nier : l'existence d'altérités sur le territoire turc. Les deux articles et leur modalité négative s'inscrivent en effet dans un dialogisme avec d'autres discours, en renvoyant involontairement à des discours préalablement tenus par les formations politiques sur les altérités ethnolinguistiques. Dans ce fonctionnement dialogique de la négation syntaxique discuté par Jacques Brès (1999), le texte législatif ne porte pas sur les réalités ethnolinguistiques ellesmêmes, mais plutôt sur les discours et les points de vue qui posent leur existence. Le texte constitue donc une sorte de «négation polémique », car il s'apparente à « une réponse, contestation d'un énoncé antérieur» (Attal, 1984, p.4), en renvoyant involontairement à des discours préalablement tenus sur 
les altérités. Les restrictions de la liberté d'expression des partis concernent également, selon l'article 81, leurs activités, pour lesquelles il prohibe l'usage de langues autres que le turc.

Si les altérités doivent être évacuées des activités des partis politiques, elles doivent encore moins figurer dans leur nom. Alors que l'on pouvait légitimement penser que l'énoncé des articles 81 et 82 était suffisamment contraignant, le législateur a tout de même pris le soin de préciser, dans l'article 96 de la même loi, « les noms de partis et les signes qui ne pourront pas être utilisés». L'article prohibe ainsi l'usage des noms, logos, surnoms et signes similaires à ceux des partis qui sont dissous définitivement par la Cour constitutionnelle ou qui restent inscrits au registre des partis politiques. De la même façon, l'article précise qu'il ne peut être fondé de partis politiques portant les noms de communiste, anarchiste, fasciste, national-socialiste, religion, langue, race, confession et région ou leurs synonymes et que «ces mots ne peuvent pas figurer dans le nom du parti ». Enfin, dans une section « Respect à Atatürk», l'article 85 énonce que les partis ne peuvent pas utiliser le nom d'Atatürk et sa photographie dans leur nom et dans leur logo.

Ainsi, si la loi sur le statut des partis politiques leur ouvre des possibilités, elle impose également des limites considérables dans leurs objectifs, discours et activités. Ces limitations sont amplifiées par l'article 10 de la loi qui prévoit une sorte de surveillance policière sur les partis. En effet, cet article énonce que le parquet du procureur de la République établit pour chaque parti politique un registre, dans lequel sont consignés la déclaration de création et ses annexes, la liste de toutes les sections, le prénom, le nom, le lieu et la date de naissance des personnes qui ont pris des responsabilités dans les organes des sections, ainsi que la liste de tous les adhérents.

La loi sur le statut des partis politiques dévoile d'une certaine façon les limites de l'exercice de la démocratie parlementaire en Turquie. À travers la loi, le législateur prend des précautions juridiques et linguistiques, restreignant la liberté d'expression et le cadre des activités des partis politiques par la menace de cette épée de Damoclès. Comme on pouvait s'y attendre, la loi a fait preuve d'une efficacité redoutable : c'est en effet sur la base de cette loi que 57 partis politiques ont été dissous dans l'histoire de la République de Turquie par la Cour constitutionnelle, le Conseil des ministres ou le Conseil national de sécurité turcs (Mert, 2008).

\section{La nomination des partis légaux kurdes}

Comment ces restrictions légales vont-elles se répercuter, sur le plan linguistique, dans le nom des partis politiques kurdes qui ont choisi de mener des activités politiques dans un cadre légal? Comme tout acte de nomination, nommer 
un parti politique implique la « représentation d'un rapport» (Siblot, 1999) à cet objet. Cette représentation, comme toutes les représentations sociales, s'inscrit dans un dialogisme, au sens de Bakhtine (1977) et d'Authier-Revuz (1995), dont l'analyse doit rendre compte pour se placer dans une perspective dynamique de la production du sens : dialogisme avec les noms des autres partis politiques par nécessité de différenciation, mais aussi dialogisme par rapport aux discours qui construisent et relayent les activités des partis.

\section{La structure du nom des partis}

La structure canonique du nom des partis est constituée d'un syntagme nominal comprenant un ou plusieurs noms communs et/ou propres et adjectifs turcs. Ils sont donc "polylexicaux» (Lecolle, 2014) et souvent descriptifs du projet sociétal des partis. La particularité des noms des partis légaux kurdes, comme ceux des partis turcs, est l'usage exclusif de parti pour désigner leur dimension politique. Le mot, qui est un emprunt français ${ }^{8}$, est défini, selon le Dictionnaire général de la langue turque, comme «ortak düşünce ve görüşteki kişilerin oluşturdukları siyasal topluluk» [communauté politique formée par les personnes partageant la même pensée et opinion]9. Cette acception du mot en turc est assez proche de celle définie par Le Petit Robert: "association des personnes unies pour défendre des intérêts, des buts communs» (2003). La loi sur le statut des partis politiques ne porte aucune obligation de l'usage du label parti dans leur nom, dont la présence récurrente s’inscrit dans la tradition politique turque, où il s'apparente à une «marque collective» (Offerlé, 2012). Le label parti bénéficie dans le discours politique turc de valeurs positives en ce qu'il transforme un ensemble d'individus en groupe social et permet aux adhérents de marquer une appartenance à la fois politique et sociale.

Deux tendances de nomination des partis kurdes se dégagent de façon contrastée : d'une part des noms de partis constitués de noms communs et d'adjectifs, et d'autre part des noms de partis intégrant des noms communs et/ou propres.

La première tendance est attestée lors de la création des premiers partis politiques. Le Halkın Emek Partisi (HEP) «Parti du travail du peuple», fondé en 1990, n'est ainsi constitué que de noms communs (halk «peuple», emek «travail »). Les autres partis créés après la dissolution du HEP et relayant sa tradition ont poursuivi cette tendance :

Demokrasi Partisi (DEP) «Parti de la démocratie»

Halkın Demokrasi Partisi (HADEP) «Parti démocratique du peuple»

8. Le mot donne lieu en turc à plusieurs dérivés : partili «membre de parti », partisiz « sans parti », partililik «fait d'être membre d'un parti », partici «partisan », partileşme « processus de formation d'un parti », partileştirme «transformer en parti».

9. Türk Dil Kurumu [Institut de la langue turque], http://www.tdk.gov.tr/index.php?option=com bts\&arama=kelime\&guid=TDK.GTS.5bge725aaeod50.90543700 (consulté le 15/10/2018). 
Cinq partis intègrent la conjonction ve «et» dans leur nom pour unir deux mots et établir un lien logique entre eux :

Barış ve Demokrasi Partisi (BDP) «Parti de la paix et de la démocratie»

Özgürlük ve Demokrasi Partisi (ÖZDEP) «Parti de la liberté et de la démocratie »

Özgürlük ve Eşitlik Partisi (ÖZEP) «Parti de la liberté et de l'égalité »

Özgürlük ve Sosyalizm Partisi (ÖSP) «Parti de la liberté et du socialisme»

Hak ve Özgürlükler Partisi (HAK-PAR) «Parti des droits et des libertés»

Comme nous allons le voir dans l'analyse des aspects lexico-sémantiques, la conjonction permet d'associer deux mots sémantiquement proches. D'autres partis utilisent une épithète (demokratik «démocratique» et katılımcı «participatif») dans le nom de leur parti :

Demokratik Halk Partisi (DEHAP) «Parti du peuple démocratique»

Demokratik Toplum Partisi (DTP) «Parti de la société démocratique»

Demokratik Bölgeler Partisi (DBP) «Parti des régions démocratiques»

Halklarin Demokratik Partisi (HDP) «Parti démocratique des peuples»

Katılımcı Demokrasi Partisi (KADEP) «Parti de la démocratie participative»

Par rapport aux noms de partis constitués de plusieurs noms et reliés par une conjonction, l'usage de l'épithète dans les noms des partis ci-dessus permet de préciser «la catégorie ou l'identité en termes d'appartenance ou de ligne politique» (Lecolle, 2014). La deuxième tendance, qui se traduit par l'usage des noms propres dans le nom des partis, est observée à partir de 2014. Il s'agit d'une période d'ouverture politique sur la question kurde en Turquie. Des militants kurdes tirent profit de cette ouverture en introduisant le toponyme Kurdistan dans le nom de trois partis politiques :

Türkiye Kürdistan Demokrat Partisi (TKDP) «Parti démocratique du Kurdistan de Turquie» Kürdistan Özgürlük Partisi (PAK) «Parti de la liberté du Kurdistan »

Kürdistan Sosyalist Partisi (PSK) «Parti socialiste du Kurdistan »

Qu'ils soient constitués de noms communs, d'adjectifs et/ou de noms propres, l'initiale du nom des partis s'écrit en majuscule, comme c'est le cas pour les noms propres en turc. Le nom de parti peut donc être considéré comme un «nom collectif» (Tournier, 1981) qui concerne un groupement humain «fondé sur un lien social et une finalité commune, un "faire ensemble" plus ou moins délimité » (Lecolle, 2014). Il s’agit de dénominations collectives désignant des personnes engagées dans un but commun et dont la construction alterne des contraintes et des possibilités. Ces dénominations résultent d'un acte de nomination, qui peut s'accomplir au moment de la déclaration de fondation d'un parti ou de la déposition de ses statuts au ministère de l'Intérieur. Conférant une existence aux partis, l'acte de nomination produit en même temps une forme de reconnaissance en tant que groupe. C'est aussi durant ce processus de nomination que le nom du parti se fixe et se fige : des syntagmes 
nominaux, incluant des noms communs et/ou propres, accèdent donc au statut de "noms propres collectifs» (Lecolle, 2014). Ce passage d'une catégorie de nom à une autre s'opère d'autant plus facilement qu'il existe un réel continuum en turc entre les noms communs et les noms propres ${ }^{10}$.

\section{Les aspects lexico-sémantiques}

Comme on vient de le voir, ce n'est que tardivement, depuis 2014, que trois partis légaux kurdes utilisent le toponyme Kurdistan dans leur nom ${ }^{11}$. Cet usage, qui n'est pas sans leur poser des problèmes judiciaires, a pour conséquence de les positionner et de les exhiber comme des partis kurdes. Cependant, en l'absence des termes Kurdes et Kurdistan, rien dans les noms des premiers partis créés à partir de 1990 ne se réfère explicitement à un quelconque engagement en faveur de la population kurde de Turquie. À l'époque des interdits frappant l'usage de ces termes dans le discours, l'implicite reste donc le moyen linguistique de détourner l'interdit et de renvoyer à une altérité en même temps qu'à un projet politique.

\section{L'implicite et le détournement de l'interdit}

Ainsi, le champ lexical des noms utilisés dans la dénomination des premiers partis légaux est constitué de halk "peuple», emek «travail», demokrasi « démocratie », demokratik « démocratique », özgürlük « liberté », eşitlik «égalité », barış « paix», sosyalizm «socialisme», hak « droit», katılımcı « participatif ». Si ces mots créent un univers de discours référant à des aspirations démocratiques, égalitaires et à des valeurs universelles des droits de l'homme, ils prennent également des significations particulières dans le contexte kurde.

C'est le cas notamment du nom halk «peuple», présent dans le nom de trois partis (HEP, HADEP, HDP). Le mot réfère à une collectivité de populations

10. Depuis la réforme de la langue turque dans les années 1930 et la volonté des autorités turques de promouvoir les noms typiquement turcs au détriment des noms provenant de langues étrangères (Szurek, 2013), des pans entiers de l'onomastique turque ont été turquifiés en intégrant des noms communs turcs. Les prénoms sont très illustratifs : Gül «rose », Birgül « une rose», Songül «dernière rose», Sema «ciel », Seda « voix». Les patronymes se sont diversifiés avec la loi nº 2525 de 1934 sur l'état civil, qui oblige les citoyens turcs à porter un nom de famille turc : şimşek «foudre», Aslan «lion», Kaplan «tigre», Arpa « orge», Sevindik « on est ravis», Bildik "on a su». Les noms communs ont été utilisés non seulement pour nommer les nouvelles localités (Akçukur: ak «blanc » çukur «fosse » «Fosse blanche», Kaleköy: kale «fort » köy «village» «Village-fort», Taşkapı : taş «pierre » kapı «porte » «Porte-pierre»), mais aussi pour renommer les lieux dont les noms référaient à des altérités ethnolinguistiques (Akin, 2017) : la commune Milikan a été renommée par Uzunçayır «Longue prairie », Holîpar Taşlıdere «Cours d'eau pierreux», Apikan par Duygulu «Sentimental», etc.

11. Les noms de partis clandestins, non soumis aux contraintes de la loi, se caractérisent par l'usage récurrent du toponyme Kurdistan : Partiya Karkarên Kurdistan (PKK) «Parti des travailleurs du Kurdistan », Kurdistan Ulusal Kurtulusçuları (KUK) «Libérateurs nationaux du Kurdistan », Partiya Yekitiya Sosyalistên Kurdistan (PYSK) «Parti de l'unité des socialistes du Kurdistan ». 
sans en préciser l'origine ethnique. Utilisé deux fois au singulier (HEP, HADEP) et une fois au pluriel (HDP), sa récurrence ne peut être expliquée que si on le replace dans la situation sociopolitique des années 1990 en Turquie. Cette période est en effet caractérisée par la poursuite de la politique officielle de déni de l'existence des Kurdes en Turquie, la guérilla armée du PKK, une politique de la «terre brûlée » et d'assassinat d'un député ${ }^{12}$, d'intellectuels, d'écrivains, de journalistes ou de simples militants politiques ${ }^{13}$. L'un des objectifs majeurs du mouvement politique kurde, légal comme clandestin, est dès lors de faire reconnaître l'existence des Kurdes. Ainsi, si le mot halk dans l'intitulé de deux premiers partis peut être considéré comme une référence aux valeurs socialistes, référence accentuée par le logo du parti, dont la forme est constituée d'une rose au centre d'un cercle de poignées de mains qui se tiennent ${ }^{14}$, il semble aussi relever d'un implicite, qui doit être lu comme Kürt halkı «peuple kurde». En effet, les publications kurdes avaient fait de ce syntagme leur cheval de bataille à la fois pour répondre au discours officiel négationniste et réclamer des droits élémentaires pour la population kurde.

Mais le mot figure au pluriel dans la dénomination du HDP «Parti démocratique des peuples ", troisième parti sur la scène politique turque après les élections législatives du 24 juin 2018 et disposant d'un groupe parlementaire à l'Assemblée nationale turque. Le mouvement légal kurde, poursuivant la tradition politique du HEP et des autres partis créés au fur et à mesure de leur dissolution par la Cour constitutionnelle, souhaitait embrasser d'autres minorités ethnolinguistiques du pays afin d'atteindre aux élections le seuil des $10 \%$ des voix indispensables pour obtenir des élus ${ }^{15}$. C'est dans cet objectif que le HDP a été fondé en 2012 comme "parti de réserve », dans l'hypothèse de la dissolution du BDP, «Parti de la paix et de la démocratie », qui est effectivement intervenue en 2014. Par son nom, le HDP s'adresse donc à toutes les altérités ethnolinguistiques de la Turquie, pour les englober dans un projet démocratique.

Le projet démocratique trouve d'ailleurs une place de prédilection dans les noms des partis. Il est exprimé par quatre occurrences de demokrasi « démocratie » et trois occurrences de demokratik « démocratique». Cette récurrence résonne comme une volonté politique de créer un véritable régime démocratique en Turquie, dans lequel la question kurde pourrait être résolue. D’autres

12. Député du DEP, M. Mehmet Sincar a été assassiné le 4 septembre 1993. «Histoire des partis politiques kurdes en Turquie », site du Parti démocratique des peuples - Représentation en Europe, http://fr.hdpeurope.com/?page_id=537 (consulté le 08/03/2019).

13. Voir La situation au Kurdistan de Turquie, Institut kurde de Paris, Bulletin de liaison et d'information, numéro spécial, novembre 1992, https://www.institutkurde.org/publications/bulletins/pdf/speciaux/nsp_turquie.pdf (consulté le 08/03/2019).

14. Voir l'article «People's Labor Party» surWikipédia, https://en.wikipedia.org/wiki/People\%27s_ Labor_Party (consulté le o8/03/2019).

15. Marie Forestier, "L'HDP, la voix des minorités turques », diffusé le 30 juin 2015 dans le journal d'Arte (durée 2 min 29 s), https://info.arte.tv/fr/lhdp-la-voix-des-minorites-turques (consulté le $08 / 03 / 2019)$. 
termes tels que özgürlük « liberté», eşitlik «égalité », barış «paix», hak «droit» sosyalizm "socialisme", utilisés dans le nom d'autres partis peuvent être lus comme des déclinaisons d'un projet démocratique fondé, selon eux, sur des revendications de liberté, d'égalité et de paix.

\section{L'émergence du Kurdistan dans le nom des partis}

Ainsi, l'implicite apparaît comme un moyen de détourner l'interdit sur le nom des partis; il permet à la fois de renvoyer à des altérités en même temps que de décrire les contours des projets politiques des partis. Cependant, selon certaines formations, il vaut mieux nommer un chat un chat et appeler les choses par leur nom. C'est dans cette entreprise que se sont lancés des partis politiques créés à partir de 2014 à la faveur de la timide démocratisation du pays et de la levée du tabou sur la question kurde.

Bravant l'interdit pour les partis d'utiliser des noms régionaux dans leurs dénominations, énoncé par l'article 96 de la loi déjà évoquée, trois partis ont ainsi utilisé le toponyme Kurdistan dans leur nom : Türkiye Kürdistan Demokrat Partisi (TKDP) «Parti démocratique du Kurdistan de Turquie»; Kürdistan Özgürlük Partisi (PAK) «Parti de la liberté du Kurdistan»; Kürdistan Sosyalist Partisi (PSK) «Parti socialiste du Kurdistan».

Il s'agit donc d'un nom propre de lieu qui désigne la région de peuplement kurde depuis que le prince seldjoukide Sandjar créa, au XIX siècle, une province du nom de Kurdistan. Deux fonctionnements caractérisent le toponyme dans le nom de ces trois partis. Le premier fonctionnement est celui qui territorialise les partis dans les régions à dominante kurde en Turquie. Ce fonctionnement du toponyme comme marqueur territorial produit un sens collectif en contexte, qui réfère en même temps aux habitants du lieu. Or, les lieux n'existent qu'occupés par des habitants; ceux-ci imprègnent les territoires de leur passage en les nommant, par leur imaginaire collectif et le rapport qu'ils entretiennent avec eux. De telle sorte que le toponyme reste imprégné des discours dans lesquels il est utilisé et qui lui assignent du sens. Comme le dit Paul Bacot, «le nom propre est en soi un discours, il est un récit qui nous parle de ce qu’il dénomme, évoquant un certain passé vu d'une certaine façon, voire un certain futur, dessinant un environnement, suggérant d'autres entités en relation avec celle qui est dénommée, esquissant sa description» (Bacot, 2010, p. 51). Si le toponyme est un discours, il est aussi un message et un projet politique. Par ce deuxième fonctionnement, le choix du toponyme prend en soi une signification politique dans le contexte conflictuel étudié. Cette signification est prépondérante par rapport à l'inscription spatiale qu'il opère. En effet, en intégrant le toponyme dans leur nom, les partis exhibent clairement leur positionnement politique; ils ont osé utiliser dans leur nom un toponyme, qui plus est longtemps frappé d’interdit, ils sont basés essentiellement dans la région kurde, 
ils s'identifient à cette région, s'appuient sur des membres kurdes, etc. Mais ils décrivent également leur projet politique, à savoir la volonté de faire reconnaître l'existence d'une altérité ethnolinguistique, d'obtenir des droits linguistiques et culturels, etc. Notons aussi que le fonctionnement du toponyme Kurdistan comme symbole de lutte s'inscrit dans un dialogisme avec le nom de partis kurdes irakien et iranien. Cela est particulièrement perceptible dans le nom du premier parti kurde, le Parti démocratique du Kurdistan de Turquie. En effet, le label Parti démocratique du Kurdistan se retrouve dans le nom des deux principaux partis kurdes irakien et iranien : le Partiya Demokrat a Kurdistana Iraqê «Parti démocratique du Kurdistan d'Irak» et le Partiya Demokrat a Kurdistana Iranê «Parti démocratique du Kurdistan d'Iran », fondés tous deux en 1946 dans l'éphémère République kurde de Mahabad en Iran (Eagleton, 1991). Après l'écrasement de la république en 1947, c'est le Parti démocratique du Kurdistan d'Irak fondé par le général Mustafa Barzani qui portera en Irak le flambeau des droits du peuple kurde. Prônant une solution politique fondée sur l'autonomie du Kurdistan irakien, le PDKI a exercé une influence politique significative sur les autres mouvements politiques kurdes. Le sigle du TKDP entre en résonance dialogique avec le sigle turc des deux partis en question (IKDP «Irak Kürdistan Demokrat Partisi » et IKDP «İran Kürdistan Demokrat Partisi »), ce qui l'inscrit dans une tradition à la fois linguistique et politique, celle de la défense de l'autonomie de la région kurde de Turquie.

\section{L'embarras du ministère de l'Intérieur face au terme Kurdistan}

Le deuxième parti, créé en 2014, s’est positionné sur la scène politique turque comme le parti qui a voulu briser le tabou officiel sur la question kurde. En introduisant et en associant à la fois les mots özgürlük « liberté » et Kürdistan dans son nom, le Parti de la liberté du Kurdistan (PAK) a posé publiquement la question de l'indépendance du Kurdistan. Contrairement aux autres partis, le PAK est en effet le seul à avoir traduit son nom en kurde. Son sigle est d'ailleurs basé sur le nom kurde du parti «Partiya Azadiya Kurdistan » et aucun sigle prenant l'écriture turque du nom (KÖP) n'est attesté.

À la différence du TKDP, dont le nom s'attache à la Turquie, le PAK n'utilise pas le toponyme Turquie dans son nom. S'inscrivant dans une perspective interkurde, le PAK prône en effet le droit des Kurdes à l'autodétermination. Ce droit n'est pas seulement revendiqué pour les Kurdes de Turquie, mais aussi pour l'ensemble des Kurdes vivant dans les autres parties du Kurdistan ${ }^{16}$.

16. Kürdistan Özgürlük Partisi Genel Başkanı Mustafa Özçelik’le Röportaj [Entretien avec le président du Parti de la liberté du Kurdistan Mustafa Özçelik], http://kurdistan-post.eu/tr/roportajlar/kurdistan-ozgurluk-partisi-genel-baskani-mustafa-ozcelikle-roportaj-hulya-yetisen (consulté le 18/10/2018). 
Comme on pouvait s'en douter, le nom et le projet politique porté par le PAK ne sont pas passés inaperçus. En effet, le PAK a annoncé sa fondation le 19 octobre 2014, et conformément à la loi sur le statut des partis politiques, remis ses statuts au ministère de l'Intérieur le 17 novembre 2014. Cependant, les fonctionnaires du ministère ont, dans un premier temps, refusé de donner l'accusé de réception qui aurait consacré officiellement sa création. Alors que selon l'article 8 de la loi sur les statuts, aucune autorisation préalable n'est requise pour la fondation d'un parti politique, le refus du ministère était fondé sur le nom du parti qu'il considérait incompatible avec la Constitution turque. Les journaux turcs se sont emparés du refus du ministère, offrant ainsi, de façon paradoxale, une forte médiatisation à la fondation du PAK. L'accusé de réception a finalement été remis aux fondateurs du PAK le 11 décembre 2014. Cependant, la Cour constitutionnelle a engagé un procès contre le parti, en mars 2015, pour violation de la loi sur son nom et ses objectifs, la Cour reprochant au PAK d'utiliser un nom kurde parallèlement à son nom turc et de défendre le droit des Kurdes à fonder un État indépendant ${ }^{17}$.

Le Parti socialiste du Kurdistan (PSK), troisième parti à utiliser le terme Kurdistan dans son nom, a été fondé en 2016. Sa création constituait une sorte de légalisation du PSK, qui existait depuis une quarantaine d'années dans la clandestinité. Les dirigeants du parti, longtemps exilés en Europe, ont pu rejoindre la Turquie à partir de 2010. Hostile à la lutte armée et prônant une solution fédérale à la question kurde en Turquie lorsqu'il était dans la clandestinité, le PSK poursuit aujourd'hui cet objectif sur le plan légal. Cependant, un procès intenté le 22 février 2019 par la Cour constitutionnelle vise la dissolution du PSK.

\section{Les sigles des partis : dialogisme et détournement}

La siglaison des noms de partis politiques est très fréquente en Turquie. Les dixsept partis auxquels nous nous sommes intéressé accompagnent systématiquement leurs noms d'un sigle. Les logos des partis intègrent d'ailleurs ces sigles avec les noms complets. La presse écrite turque réfère aux partis uniquement à travers leurs sigles ${ }^{18}$. C'est dire la notoriété et la fonction d'identification des sigles de partis dans le discours ${ }^{19}$. Dotés d'une «fonction dénominative lapidaire véhiculaire» (Percebois, 2001, p. 629), les sigles résultent du processus d'abréviation qui procède d'une nécessité d'économie linguistique et de mobilisation et de communication (Bacot etal., 2011). Dans le cas des noms des partis

17. Yargıtay'dan Pak'a “Kürtçe” Uyarısı [Avertissement de la Cour de cassation au Pak au sujet du «kurde»], https://www.haberler.com/yargitay-dan-pak-a-kurtce-uyarisi-7053405-haberi/ (consulté le 18/10/2018).

18. Demirtaş ve Önder'e hapis cezası [Peine de prison à Demirtaş et Önder], Cnnturk, https://www. cnnturk.com/turkiye/demirtas-ve-ondere-hapis-cezasi (consulté le 18/10/2018).

19. Pour une discussion des usages des sigles, voir le numéro 95 de la revue Mots consacré aux "Sigles et acronymes en politiques» (2011). 
kurdes, outre la fonction d'identification qu'ils assurent, les sigles constituent également un moyen efficace d'établir le dialogisme et de détourner l'interdit.

En effet, les sigles des dix premiers partis kurdes semblent s'inscrire dans une certaine régularité : HEP, DEP, ÖZDEP, ÖZEP, HADEP, DEHAP, DTP, DBP, BDP, HDP. Ces partis incarnent la même tradition politique et, comme nous l'avons vu ci-dessus, ont été créés l'un après l'autre au fur et à mesure que l'un d'eux était dissous par la Cour constitutionnelle. À part DBP et HDP, tous les autres partis ont été dissous et un procès est en cours contre le HDP. Il est possible de répartir les sigles de partis en trois groupes proches sur le plan lexico-sémantique:

HEP Halkın Emek Partisi « Parti du travail du peuple»

HADEP Halkın Demokrasi Partisi «Parti démocratique du peuple»

DEHAP Demokratik Halk Partisi «Parti du peuple démocratique»

HDP Halklarin Demokratik Partisi « Parti démocratique des peuples»

ÖZDEP Özgürlük ve Demokrasi Partisi «Parti de la liberté et de la démocratie»

ÖZEP Özgürlük ve Eşitlik Partisi «Parti de la liberté et de l'égalité»

DEP Demokrasi Partisi «Parti de la démocratie»

DTP Demokratik Toplum Partisi «Parti de la société démocratique»

DBP Demokratik Bölgeler Partisi «Parti des régions démocratiques»

BDP Barışve Demokrasi Partisi «Parti pour la paix et la démocratie»

Le premier groupe se caractérise par la récurrence du mot halk «peuple» et du qualificatif demokratik « démocratique». Le mot özgürlük « liberté » caractérise le deuxième groupe, alors que le troisième groupe est dominé par la présence de trois occurrences de demokratik et d'une occurrence de demokrasi. Si les différents noms de parti relèvent de l'article 96 de la loi sur les statuts des partis, qui prohibe la reprise du nom d'un parti dissous, les sigles permettent d'établir un dialogisme orienté vers le nom des anciens partis dissous, et ce malgré la distance temporelle qui sépare leur fondation et leur dissolution. Ils constituent des messages politiques à la fois aux autorités turques et aux électeurs pour leur signifier la permanence d'une tradition politique.

Un autre fonctionnement des sigles est celui qui consiste à contourner l'interdit sur le nom des partis, la loi portant sur les seuls signifiants graphiques. Ce fonctionnement est attesté dans le sigle de Katılımcı Demokrasi Partisi «Parti de la démocratie participative», qui est KADEP. Le parti a été fondé en 2006, à un moment où il n'était pas envisageable d'utiliser le toponyme Kurdistan. Cependant, le nom se prononce en turc de la façon suivante : [ka-de-pe]. Cette prononciation du sigle est identique à celle du KDP « Kürdistan Demokrat Partisi », dont nous avons vu qu'il incarne une tradition politique initiée en 1946. Le [k] du katılımcı «participatif» sert de substitut au [k] du Kurdistan. Le fonctionnement du sigle peut être considéré ici comme un «détournement dévoilant» qui fait « appel à des références idéologiques et à un contexte historique » (Renard, 2011, p.36). En référant à une tradition politique, le sigle KADEP, par son contenu oral, exhibe donc un autre contenu politique que celui qu'il exprime littéralement. 
Les noms des partis légaux kurdes en Turquie reflètent donc les évolutions du pays sur la question kurde et les projets de chacun des partis concernant les solutions à cette question. L'interdit sur l'usage des termes Kurde et Kurdistan dans le nom des partis politiques les a conduits à faire preuve d'imagination pour faire référence à une altérité déniée en même temps qu'à un projet politique. L'implicite s'est dès lors révélé comme un moyen efficace de contourner les restrictions légales. La levée progressive de l'interdit s'est répercutée dans le nom de trois partis, dans lesquels le toponyme Kurdistan figure en dépit des procès en dissolution visant deux d'entre eux. Nos analyses montrent comment le nom de parti apparaît lui-même comme un message politique décrivant les objectifs des partis : démocratisation du pays, aspiration à la liberté, à la paix, ou à l'égalité kurde-turque, recherche d'autonomie ou d'indépendance de la région kurde, les noms de partis résonnent comme autant de projets politiques.

\section{Références}

AKIN Salih, 2017, «Restitution des toponymes kurdes en Turquie», dans Les langues et leurs territoires : entre conflit et cohabitation, M. Dokthourichvili, J. Boissonneault et A. Reguigui éd., Sudbury (Ontario), Université Laurentienne, Département d'études françaises, p. 513-534.

- 1996, "Les mouvements de jeunesse et la formation des élites kurdes», Agora débats/jeunesses, no6, p.111-122.

- 1995, Désignation du peuple, du territoire et de la langue kurdes dans le discours scientifique et politique turc, thèse de doctorat sous la direction de B. Gardin, Rouen, Université de Rouen-Normandie.

AtTAL Pierre, 1984, «Deux niveaux de négation », Langue française, n6 62, p. 4-11.

AUtHIER-REVUz Jacqueline, 1995, Ces mots qui ne vont pas de soi : boucles réflexives et non-coïncidences du dire, Paris, Larousse, 2 vol.

BAсот Paul, 2010, «Développement et diversification d'une onomastique politique», Mots. Les langages du politique, n 94, p. 47-56.

Bacot Paul, Desmarchelier Dominique, Honoré Jean-Paul, 2011, «Les usages politiques d'une réduction », Mots. Les langages du politique, nº 95, p. 5-10.

BAKHTINE Mikhail, 1977, Le marxisme et la philosophie du langage : essai d'application de la méthode sociologique en linguistique, M. Yaguello trad., Paris, Minuit.

BozArs LAN Hamit, 1997, La question kurde : États et minorités au Moyen-Orient, Paris, Presses de Sciences Po.

BRES Jacques, 1999, «Vous les entendez? Analyse du discours et dialogisme », Modèles linguistiques, $\mathrm{n}^{\circ} 40, \mathrm{p} .71-86$.

DEREN Seçil, 2005, «Le kémalisme aujourd'hui », Outre-Terre, nº 10, p. 145-156.

DuFour Françoise, 2011, «Le sigle comme modalité de dissociation énonciative. Le cas des alternances d'usage PMA / Pays les moins avancés », Mots. Les langages du politique, n०05, p. $75-87$. 
EAgLETon William, 1991, La république kurde de 1946, C. Ter-Sarkissian trad., Bruxelles, Éditions Complexe.

LECOLLE Michelle, 2014, «Dénomination de groupes sociaux : approche sémantique et discursive d'une catégorie de noms propres ", dans $4^{e}$ Congrès mondial de linguistique française, F. Neveu, P. Blumenthal, L. Hriba et al. éd., Les Ulis, EDP Sciences, p. 2265-2281, http://dx.doi.org/10.1051/shsconf/20140801063 (consulté le 8 mars 2019).

Mert Yener Lütfü, 2008, Cumhuriyet Döneminde Kapatılan Siyasi Partiler : Kapatma Davaları Gerekçeleri ve Sonuçları, Ankara, İlkim.

OfFERLÉ Michel, 2012, Les partis politiques, Paris, PUF.

PERCEBoIs Jacqueline, 2001, "Fonctions et vie des sigles et acronymes en contextes de langues anglaise et française de spécialité», Meta. Journal des traducteurs, vol.XLVI, no ${ }^{4}$, p. $627-645$.

RENARD Jean-Bruno, 2011, "Le détournement de sigles. Entre jeu de mots et expression contestataire», Mots. Les langages du politique, n 95, p. $29-42$.

Schmid Dorothée, 2017, "Turquie : du kémalisme au néo-ottomanisme», Questions internationales, n०87, p. 105-111.

SIB LOT Paul, 2007, «Problématique de la nomination : du répertoire des sens à l'analyse de leur production », Neologica. Revue internationale de néologie, nº 1, p. 33-48.

- 1999, «Appeler les choses par leur nom. Problématique du nom, de la nomination et des renominations", dans Noms et re-noms : la dénomination des personnes, des populations, des langues et des territoires, S. Akin éd., Mont-Saint-Aignan, Publications de l'université de Rouen, p.11-31.

Skutnabb-KAngas Tova, Phillipson Robert, 1999, "Linguistic genocide and human rights: Kurdish considerations», dans The Kurds: Perspectives on an Unique Culture, P. Sundqvist éd., J. O’Neill trad., Helsinki, Suomen Rauhanliitto, YK-Yhdistys, p. 25-48.

SZUREK Emmanuel, 2013, "Appeler les Turcs par leur nom. Le nationalisme patronymique dans la Turquie des années 1930 ", Revue d'histoire moderne \& contemporaine, no6o-2, p. 18-37.

TOURNIER Maurice, 1981, "Vers une grammaire des dénominations socio-politiques au début de la Troisième République (1879-1905)», Mots, n² 2, p. 51-72.

YILMAz Özcan, 2013, La formation de la nation kurde en Turquie, Paris, Genève, PUF, Graduate Institute Publications. 


\section{Résumé / Abstract / Compendio}

\section{Noms de partis et messages politiques : le cas des partis politiques kurdes légaux en Turquie}

Cet article se propose d'examiner les noms des partis politiques kurdes légaux en Turquie. Le corpus d'études est constitué du nom de dix-sept partis légaux fondés entre 1990 et 2014. L'examen de la structure formelle et lexico-sémantique des noms de partis est articulé aux contraintes légales sur la dénomination des partis, telles qu'elles sont imposées par la loi no 2820 sur le statut des partis politiques en Turquie. Les analyses montrent comment les noms des partis politiques kurdes reflètent les évolutions du pays sur la question kurde et les projets de chacun des partis concernant les solutions à cette question : démocratisation du pays, aspiration à la liberté, à la paix, ou à l'égalité kurde-turque, recherche d'autonomie ou d'indépendance de la région kurde, les noms de partis résonnent comme autant de messages politiques.

Mots-clés : nom de parti, dialogisme, implicite, kurde, Turquie

\section{Party names and political messages: the case of legal Kurdish political par- ties in Turkey}

This article aims to examine the names of Kurdish political parties in Turkey. The corpus of studies is made up of the names of seventeen legal parties that were founded between 1990 and 2014. The examination of the formal and lexical-semantic structure of party names is articulated with the legal constraints on the naming of parties, as imposed by Law No. 2820 on the status of political parties in Turkey. The analyses show how the names of Kurdish political parties reflect the country's evolution on the Kurdish issue and the projects of each party concerning the solutions to this issue: whether it be democratization of the country, aspiration for freedom, peace, or Kurdish-Turkish equality, search for autonomy or independence of the Kurdish region, the party's name sounds as a political message.

Keywords: party name, dialogism, implicit, Kurdish, Turkey

\section{Nombres de partidos y mensajes políticos: el caso de los partidos políticos legales kurdos en Turquía}

Este artículo se propone examinar los nombres de los partidos políticos legales kurdos en Turquía. El corpus del estudio está constituido por diecisiete partidos legales fundados entre 1990 y 2014. El examen de la estructura formal y léxico-semántica de los nombres de los partidos estan relacionados con los límites legales sobre la dominación de los partidos, tales como fueron impuestos por la ley $\mathrm{N}^{\circ} 2820$ sobre el estatuto de los partidos políticos en Turquía. Los análisis realizados muestran cómo los nombres de los partidos políticos kurdos reflejan la evolución del país sobre la situación kurda y los proyectos de cada uno de ellos en lo que concierne las soluciones de esta cuestión: democratización del país, aspiración a la libertad, a la paz, o a la igualdad kurda y turca, búsqueda de la autonomía o de la independencia de la región kurda, el nombre del partido resuena tanto como lo hace el mensaje político.

Palabras claves: nombre de los partidos, dialogismo, implícito, kurdo en Turquía 